\title{
Medida del emprendizaje: adaptación y validación de la escala de actitudes emprendedoras EASS en profesores españoles
}

\section{Measurement of entrepreneurship/learning: adaptation and validation of the scale of entrepreneurship attitudes EASS in spanish teachers}

\author{
Oliver A. ${ }^{1 *}$ Galiana $L,{ }^{1}$ Calatayud $P,{ }^{1}$ Piacentini-Genovart $D,{ }^{1}$.
}

\begin{abstract}
${ }^{1}$ Universitat de València, Facultad de Psicología, Departamento de Metodología de las Ciencias del Comportamiento, España. Av. Blasco Ibañez, 21, Valencia, 46020. Correspondencia: oliver@uv.es
\end{abstract}

Recibido: 15-01-2016; Aceptado: 28-03-2016

\section{Resumen.}

El objetivo de este trabajo es ofrecer un instrumento de evaluación que permita conocer las actitudes emprendedoras del profesorado, mediante la adaptación de la original Escala de Actitudes Emprendedoras para Estudiantes, al tiempo que facilitar una primera aproximación a su diagnóstico en el ámbito español, con proyección en el área de habla hispana. En el marco de un diseño transversal, se llevó a cabo una encuesta on-line en un único momento temporal en la que participaron 155 profesionales de la educación. El instrumento adaptado (Escala de Actitudes Emprendedoras para Profesores, EAST), basado en la Escala de Actitudes Emprendedoras para Estudiantes de Oliver y Galiana (2016), evalúa 6 actitudes emprendedoras con 18 ítems: proactividad, ética profesional, empatía, innovación, autonomía y toma de riesgos, que definen a la persona con motivación hacia el emprendimiento. El análisis factorial confirmatorio ofrece resultados adecuados: $\mathrm{X}^{2}(120)=214.225(p<.001), \mathrm{CFI}=$ $.963, \mathrm{TLI}=.953, \mathrm{SRMR}=.011, \mathrm{RMSEA}=.071$. Se obtienen estimaciones de fiabilidad excelentes, con alfas de Cronbach de .95 a .97 para las seis dimensiones. Se detecta una relación negativa del emprendimiento con la edad, y una mayor presencia en mujeres. Los resultados muestran unas propiedades psicométricas adecuadas de la nueva adaptación, siendo este, hasta donde se conoce, el primero desarrollado para evaluar en educadores actitudes emprendedoras en el contexto hispano-hablante.

Palabras clave: emprendimiento, profesores, educación, instrumento de medida, adaptación, modelos de ecuaciones estructurales, análisis factorial confirmatorio. 


\section{Abstract.}

The aim of this study is to offer an assessment instrument to know the entrepreneurial attitudes of the professorate or educators (EAST), adapted from the Entrepreneurial Attitudes Scale for Students (EASS), and also to provide a first approximation to diagnosis in the field of Spanish-speaking context. A cross-sectional on-line survey was carried out in a single time, in which 155 teachers participated. The new adapted instrument, based on the Entrepreneurial Attitudes Scale for Students (EASS), assesses six entrepreneurial attitudes: proactivity, professional ethics, empathy, innovation, autonomy, and risk taking that define the individual with motivation towards entrepreneurship. Confirmatory factor analysis provides appropriate results: $X 2(120)=214.225(p<.001)$, CFI $=.963$, TLI $=.953$, SRMR $=.011$, RMSEA $=$ .071. Reliability estimations are also calculated, with Cronbach's alphas ranging from .95 to .97 for the six dimensions. A negative relation regarding entrepreneurship and age is found, as well as higher levels of entrepreneurial attitudes in women than in men. Results show appropriate psychometric properties. As far as literature has been revised, the adaptation presented here is the only developed for this purpose in Spanish-speaking context so far.

Key words: entrepreneurship, teachers, education, measurement instrument, adaptation, structural equation models, confirmatory factor analysis.

\section{Introducción}

Que el emprendimiento es pieza clave para la creación de empleo y el desarrollo económico es una visión que la sociedad actual comparte y que los medios de comunicación reflejan cada día. Los programas y cursos centrados en el emprendimiento empiezan a ser algo habitual en las instituciones educativas superiores, que atraen cada vez más el interés de políticos y gestores (Gibb y Hannon, 2006), pues se espera que las instituciones educativas enseñen competencias emprendedoras (OECD, 2009). Claro ejemplo de este interés es la publicación reciente de "Entrepreneurship Education at School in Europe National Strategies, Curricula and Learning Outcomes" (Comisión Europea, 2012a), documento en el que se remarca la importancia del que se ha acuñado como "emprendizaje" o educación en emprendimiento. También en nuestro contexto más cercano, el español, se han llevado a cabo actuaciones para promover el emprendimiento en la educación, como la Estrategia Juventud 2020 o el Plan de Acción 2014-2016, que plantean la educación y formación, así como el empleo y emprendimiento, como principales ejes estratégicos. De esta forma, la educación en emprendimiento ha trascendido los límites de las escuelas de negocios y ahora se enseña en todo tipo de instituciones educativas, a estudiantes con todo tipo de formaciones curriculares (Blenker, Elmholdt, Frederiksen, Korsgaard y Wagner, 2014).

Este nuevo escenario ha creado la necesidad de investigar sobre la educación en emprendimiento (Pittaway y Cope, 2007). De hecho, la literatura de la última década ha mostrado claras relaciones entre emprendimiento y educación (Mars y Ríos-Aguilar, 2010). Su principal caldo de cultivo es el apoyo de los profesores, la escuela y la educación (Gur-Erdogan, Eksioglu, Zafer-Gunes y Sezen-Gultekin, 2014). Por ello, es importante que los profesores sean capaces de crear ambientes que faciliten el emprendimiento, y que las familias complementen este proceso (Eraslan, 2011). Leisey y Lavaroni (2000), por ejemplo, encontraron evidencia de cómo el uso de historias de vida de 
personas emprendedoras mejoraba las actitudes hacia el emprendimiento de sus estudiantes.

Teske y Williamson (2006) definieron a los emprendedores en educación como personas que buscan promover cambios en el sistema de educación pública para transformar la forma en que se educa. Borasi y Finnigan (2010) estudiaron diversas estrategias de emprendimiento que los profesores utilizaron para mejorar su institución. Con el mismo objetivo, Eyal y Yosef-Hassidim (2012) analizaron las distintas formas que los profesores utilizaban para llevar a cabo iniciativas emprendedoras e innovadoras para mejorar sus escuelas. En esta misma línea, diversos estudios han investigado la innovación en lo personal de los futuros docentes (Atali y Sertbas, 2013; Bayraktar, 2012; Celik, 2013; Gur-Erdogan y Zafer-Gunes, 2013) y sus características emprendedoras (Akyurek y Sahin, 2013), esperando que los profesores $y$ los futuros docentes enseñen a los emprendedores de la futura generación y que sean ellos mismos emprendedores y personas innovadoras (Gur-Erdogan et al., 2014).

Sin embargo, y tal y como ha apuntado una revisión reciente de la literatura, se requiere mayor evidencia cuantitativa. En muchos de estos estudios hay predominancia de métodos cualitativos (Blenker et al., 2014), también en nuestro entorno más próximo (Benavides, Sánchez y Luna, 2004), y se detecta un uso de técnicas cuantitativas limitado tan solo a lo descriptivo. Un problema añadido es la falta de instrumentos disponibles para la medida de estas actitudes emprendedoras del profesorado. Ante esta inexistencia de medidas, Oliver y Galiana (2015) desarrollaron un modelo de medida de 18 items tipo Likert basado en la existencia de seis dimensiones claves en las actitud emprendedora: proactividad, ética profesional, empatía, innovación, autonomía y toma de riesgos. La proactividad se ha definido como "una perspectiva de futuro acompañada de actividades innovadoras o nuevas aventuras" (Lumpkin y Dess, 1996, p. 146). La proactividad en emprendedores debe entenderse como iniciativa y planificación a largo plazo; profesores y estudiantes con esta actitud pueden identificar y explotar oportunidades (Dyson, 2001). La ética profesional, por su parte, se refiere a tener una fuerte ética de trabajo, y lleva tiempo documentada su relación con la práctica empresarial (Schafermeyer y Hobson, 1997). La empatía, se entiende en el modelo de estas dos autoras, como la capacidad de comprender los sentimientos de otra persona. Es importante para un educador emprendedor la capacidad de empatizar con otras personas, la creencia de contribuir a la sociedad y el deseo de ayudar a otros (Bonnarens, 1996; Miner, 1997).

La innovación se refiere a la "tendencia de participar y apoyar ideas novedosas, ensayos y procesos creativos que pueden dar lugar a nuevos productos, servicios o conocimientos tecnológicos" (Lumpkin y Dess, 1996, p. 142), y es una de las actitudes que se han aplicado tradicionalmente a las empresas siendo ahora fácilmente extrapolables a las personas emprendedoras, tanto dentro como fuera del contexto educativo. La autonomía se define como la capacidad de la persona para darse reglas a sí misma o decidir y actuar sin intervención ni influencia externa, no permitiéndose organizaciones superiores (Lumpkin y Dess, 1996). Finalmente, la toma de riesgos, indica que algunos individuos se sienten cómodos con este y especialmente se muestran seguros en sus propias habilidades (Evans y Jovanovic, 1989; Puri y Robinson, 2005).

Dado lo anteriormente expuesto, el objetivo de este trabajo es doble: 1) Ofrecer un instrumento de evaluación basado en la adaptación del cuestionario desarrollado por las propias autoras en el ámbito de los estudiantes de 
secundaria y universitarios (Oliver y Galiana, 2015) que permita conocer las actitudes emprendedoras de los educadores hispano-hablantes, como potencial agente en la promoción del emprendimiento en sus educandos, 2) Facilitar una primera aproximación empírica al contexto español de los profesores emprendedores, ofreciendo información sobre la presencia de dichas actitudes de "emprendizaje" tanto en profesores en ejercicio como en formación.

\section{Metodología}

\section{Diseño, procedimiento y muestra.}

El presente estudio tiene un diseño de investigación transversal en único momento temporal. Se llevó a cabo una encuesta on-line sobre tres colectivos de la provincia de Valencia, España: 1) a profesores universitarios de Psicología, Actividad Física y Deporte, Ciencias de la Educación de la Universitat de Valencia (UV) y la Universidad Católica de Valencia (UPV), respectivamente pública y privada; 2) a profesores de secundaria de centros públicos, privados y concertados y 3) a futuros profesores ya graduados cursando máster de educación secundaria en las dos universidades que ofrecen la titulación (de nuevo UV y UCV). Para ello, se utilizó la plataforma Survey Monkey, que cumple con los requisitos exigibles de confidencialidad y anonimato.

El contacto con los educadores actuales y futuros, se realizó a través de diversos centros educativos; se consiguieron los correspondientes permisos y garantizando el anonimato y la confidencialidad de los participantes. Los centros intervinientes remitieron a las direcciones de e-mail de sus docentes $\mathrm{y} / \mathrm{o}$ estudiantes de educación la invitación a participar y el enlace a la encuesta. El tiempo estimado de cumplimentación fue de 15 minutos.

La muestra total fue de 155 profesores. No se dispone de tasa de respuesta, pues los centros participantes únicamente accedieron a facilitar la invitación a participar con enlace a la encuesta, pero no ofrecieron datos sobre el número de destinatarios de sus listas de correo. De los participantes, un $62.3 \%$ son hombres y un $37.7 \%$ mujeres. La media de edad es de 36.14 años (DT=13.56). En cuanto a si están en activo o preparándose para ser docentes, un $52.9 \%$ trabajaba como profesor en el momento de la encuesta y un $47.1 \%$ está preparándose para hacerlo en un futuro próximo. Un $53.3 \%$ trabajaba o estudiaba en un centro público, un $40.0 \%$ en uno privado, y el $6.7 \%$ restante en uno concertado. Para el tratamiento estadístico, y dado el número limitado de participantes en algunas categorías; se procedió a recodificar la variable únicamente en dos agrupaciones, público $(53.3 \%)$ vs. privado/concertado $(46.7 \%)$. Respecto al nivel educativo, el $1.3 \%$ centra su actividad docente actual o futura en Educación Infantil, un 1.3\% en Educación Primaria, un $46.7 \%$ en Educación Secundaria y un $50.7 \%$ en Educación Universitaria. En la misma línea, se utilizaron tan solo las dos últimas categorías (Educación Secundaria y Educación Universitaria) al analizar la variable nivel educativo.

Instrumento. La nueva adaptación que se presenta, la escala de actitudes emprendedoras en educadores, o profesores, como se puede consultar en el anexo, consiste en la adaptación al contexto de profesores de la Escala de Actitudes Emprendedoras para Estudiantes EASS (Olivery Galiana, 2015). Esta adaptación se realizó proponiendo a un grupo de 4 investigadores en psicología (de ámbitos educativo, organizacional y de metodología), redactados alternativos en aquellos ítems que lo requerían pues hablaban de emprendimiento educativo circunscrito a estudiantes. De esos ítems, se escogió para su validación los redactados de consenso, que aparecen en el listado como 9, 11, 12, 13, 16 y 17 . En ninguno de ellos se detectaron problemas de comprensión, menor tasa de respuesta - mal funcionamiento. Ambas escalas evalúan, mediante 18 ítems, 6 actitudes claves hacia el emprendimiento: 
proactividad, ética profesional, empatía, innovación, autonomía y toma de riesgos y en el caso de ítems de proactividad y ética profesional; no fue necesario adaptar ningún enunciado, se mantuvieron los ítems en su formulación original. Todas estas actitudes, recogidas de la literatura tradicional (Evans y Jovanovic, 1989; Lumpkin y Dess, 1996; Miller, 1983, Schafermeyer y Hobson, 1997) y complementadas con aportaciones más recientes (Hermansen-Kobulnicky, 2004), son capaces de definir a la persona con motivación hacia el emprendimiento, ya sea estudiante o profesor.

Análisis de datos. En primer lugar, y para evaluar la validez de la escala, se estima y evalúa un modelo de ecuaciones estructurales, en concreto, un análisis factorial confirmatorio, en el que se plantean por hipótesis 6 factores latentes, correspondientes a las 6 actitudes emprendedoras, correlacionados entre sí. El método de estimación es máxima verosimilitud con correcciones robustas. Para evaluar el modelo, se utilizan diversos índices de ajuste, tal y como recomienda la literatura (Tanaka, 1993): el estadístico chi-cuadrado (Kline, 1998); CFI y TLI, que con valores mayores de .90 e, idealmente, mayores de .95, indican un ajuste adecuado del modelo (Hu y Bentler, 1999); y SRMR y RMSEA, que se consideran apropiados cuando toman valores inferiores a .05 y .08, respectivamente (Steiger y Lind, 1980). El modelo fue estimado mediante el programa Mplus. Además, se evaluó también el ajuste analítico del modelo, a partir de las saturaciones factoriales de los ítems.

Una vez estudiada la validez factorial, se evalúa la fiabilidad de la escala. Para ello, se calculan alfas de Cronbach para cada una de las dimensiones o factores de las actitudes emprendedoras. El alfa de Cronbach es el índice más utilizado por la comunidad científica para evaluar la fiabilidad de las escalas, con valores de .70 a .79 considerados moderados, y estimaciones de .80 o superiores, interpretadas como alta fiabilidad (Cicchetti, 1994; Clark y Watson, 1995). Finalmente, se comparan los resultados en las actitudes emprendedoras en función de grupos y se estudia su relación con la edad. Para ello, se obtienen correlaciones de Pearson con la variable edad y se realizan cuatro análisis de varianza multivariados (MANOVA), teniendo como variables dependientes las seis actitudes emprendedoras y como independientes el sexo, la condición de docente (en ejercicio o en formación), tipo de centro (público o privado/concertado) y el nivel educativo en el que se trabaja como docente (infantil, primaria, secundaria, universidad). Dado que la edad muestra una relación estadísticamente significativa con las actitudes emprendedoras, pasa a emplearse como covariable en los distintos MANOVA realizados (esto es, se estudia el efecto de las variables independientes sexo, condición del docente, tipo de centro y nivel educativo, controlando porel posible efecto de la edad). Se aporta la traza de Pillai, el índice más robusto frente al incumplimiento de supuestos (Tabachnick y Fidell, 2007). Si la prueba $F$ resulta estadísticamente significativa, se realizan pruebas a posteriori de Bonferroni para las diferencias entre grupos, para determinar qué grupos difieren significativamente en medias. El tamaño del efecto se evalúa mediante eta-cuadrado parcial, con rationale de $.02, .13$ y .26 para tamaños del efecto pequeños, medios y grandes, respectivamente (Cohen, 1992).

\section{Resultados}

Validación de la nueva adaptación: escala de actitudes emprendedoras en educadores. El análisis factorial confirmatorio muestra un ajuste general adecuado: $X 2(120)=214.225$ $(p<.001)$, CFI $=.963$, TLI $=.953$, SRMR $=.011, \mathrm{RMSEA}=.071$ (intervalo de confianza al $95 \%=.055-.086)$. El ajuste analítico también es apropiado, con saturaciones factoriales elevadas y estadísticamente significativas para todos los ítems, tal y como se puede 
observar en la figura 1, y entre todas las dimensiones, como se ve en la tabla 1.

Figura 1.Saturaciones factoriales del análisis factorial confirmatorio propuesto para la nueva adaptación midiendo actitudes emprendedoras en educadores.

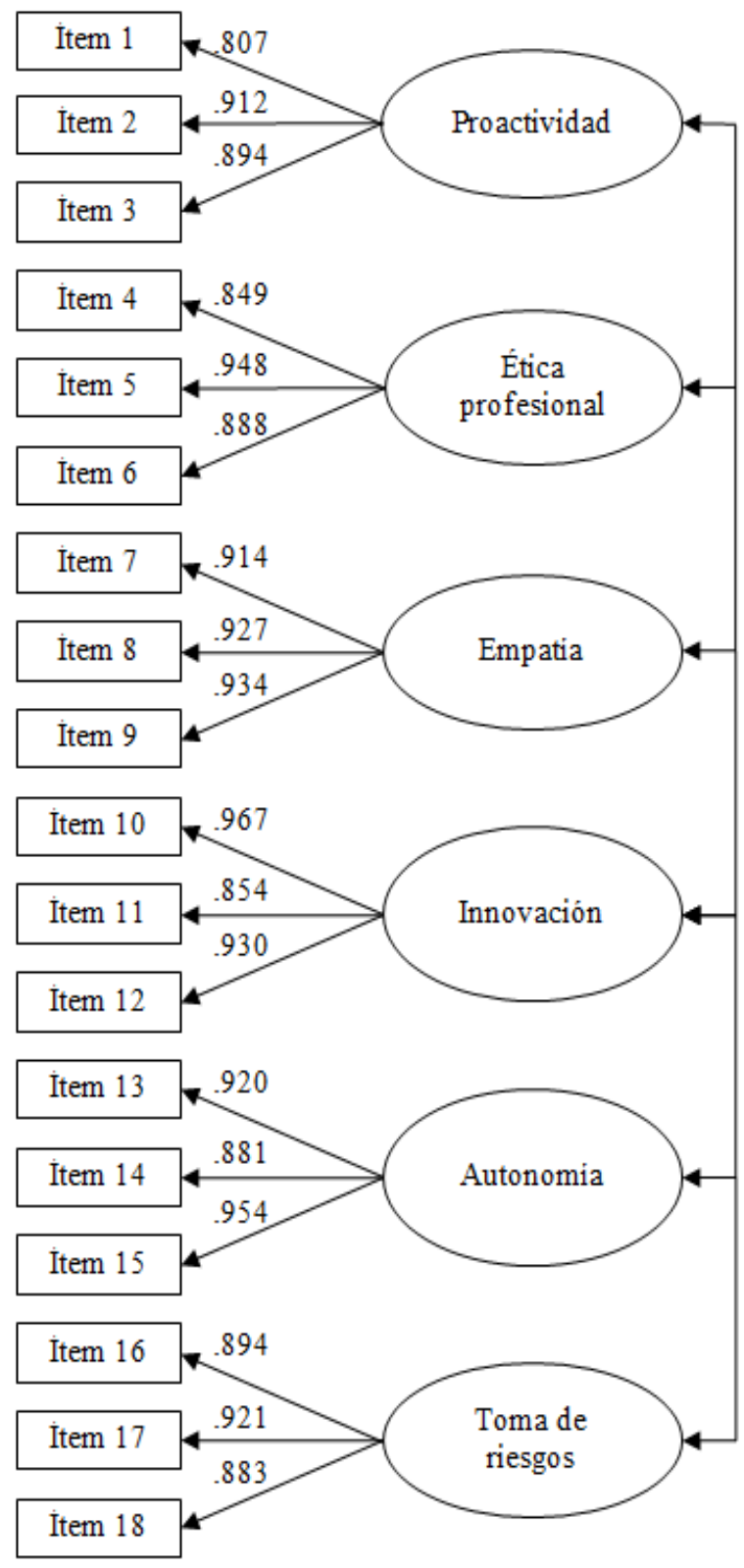

Nota: Todas las saturaciones factoriales estadísticamente significativas $(p<.05)$, errores estándar y correlaciones entre factores pueden consultarse en la tabla
Tabla 1. Correlaciones entre los factores del análisis factorial confirmatorio.

\begin{tabular}{|c|c|c|c|c|c|}
\hline & PR & EP & $\mathbf{E}$ & IN & AU \\
\hline Proactividad & -- & & & & \\
\hline Ética profesional & .996 & -- & & & \\
\hline Empatía & .979 & .992 & -- & & \\
\hline Innovación & .987 & .985 & .982 & -- & \\
\hline Autonomía & .998 & .994 & .982 & .976 & -- \\
\hline Toma de riesgos & .960 & .967 & .952 & .976 & .974 \\
\hline
\end{tabular}

En cuanto a la fiabilidad, alfas de Cronbach de .95 para la dimensión de proactividad, .96 para ética profesional, .97 para empatía, .97 para innovación, .97 para autonomía y .96 para toma de riesgos.

Análisis diferencial por grupos. Las correlaciones entre la edad y las actitudes emprendedoras son todas estadísticamente significativas y negativas, identificándose menor puntuación en actitudes emprendedoras a mayor edad de los docentes. Estas correlaciones pueden verse en detalle en la tabla 2.

Tabla 2. Correlaciones entre la edad y las actitudes emprendedoras.

$\begin{array}{cc} & \text { Edad } \\ \text { Proactividad } & -.433 \\ \text { Ética profesional } & -.414 \\ \text { Empatía } & -.376 \\ \text { Innovación } & -.400 \\ \text { Autonomía } & -.425 \\ \text { Toma de riesgos } & -.364\end{array}$

Nota: Todas las correlaciones estadísticamente significativas $(p<0.01)$. Fuente: Elaboración propia.

Finalmente, los resultados de los MANOVA muestran relación significativa entre las actitudes emprendedoras y el $\operatorname{sexo}\left(F(6,136)=2.253, p=.042, \eta^{2}=\right.$ $.090)$, pero no entre estas y condición docente -si los docentes estaban trabajando o no- $(F(6,136)=0.954$, 
$\left.p=.002, \eta^{2}=.040\right)$, el tipo de centro $\left(F(6,133)=0.837, p=.544, \eta^{2}=\right.$ .036) y el nivel educativo en el que se trabaja o estudia $(F(6,133)=1.971$, $\left.p=.074, \eta^{2}=.082\right)$. Los ANOVA de continuación en función del sexo, que se pueden observar en la tabla 3 , indican diferencias significativas en todas las actitudes emprendedoras.

Tabla 3. ANOVA de continuación

\begin{tabular}{ccccccc}
\hline $\begin{array}{c}\text { Fuente de } \\
\text { variación }\end{array}$ & $\begin{array}{c}\text { Variables } \\
\text { dependientes }\end{array}$ & $\begin{array}{c}\text { gl } \\
\text { efecto }\end{array}$ & $\begin{array}{c}\text { gl } \\
\text { error }\end{array}$ & $\boldsymbol{F}$ & $\boldsymbol{p}$ & $\eta^{2}{ }_{\text {parcial }}$ \\
\hline Sexo & $\begin{array}{c}\text { Proactividad } \\
\text { Ética } \\
\text { profesional }\end{array}$ & 1 & 141 & 9.244 & .003 & .062 \\
& Empatía & 1 & 141 & 9.247 & .003 & .062 \\
& $\begin{array}{c}\text { Innovación } \\
\text { Autonomía }\end{array}$ & 1 & 141 & 8.052 & .005 & .054 \\
& $\begin{array}{c}\text { Toma de ri- } \\
\text { esgos }\end{array}$ & 1 & 141 & 9.704 & .002 & .064 \\
\hline Fuente: Elaboración propia. & & & & .063 & .015 & .041 \\
\hline
\end{tabular}

En todos los casos, estas diferencias fueron a favor de las mujeres, con mayores medias en las actitudes emprendedoras, tal y como se muestra en la figura 2.

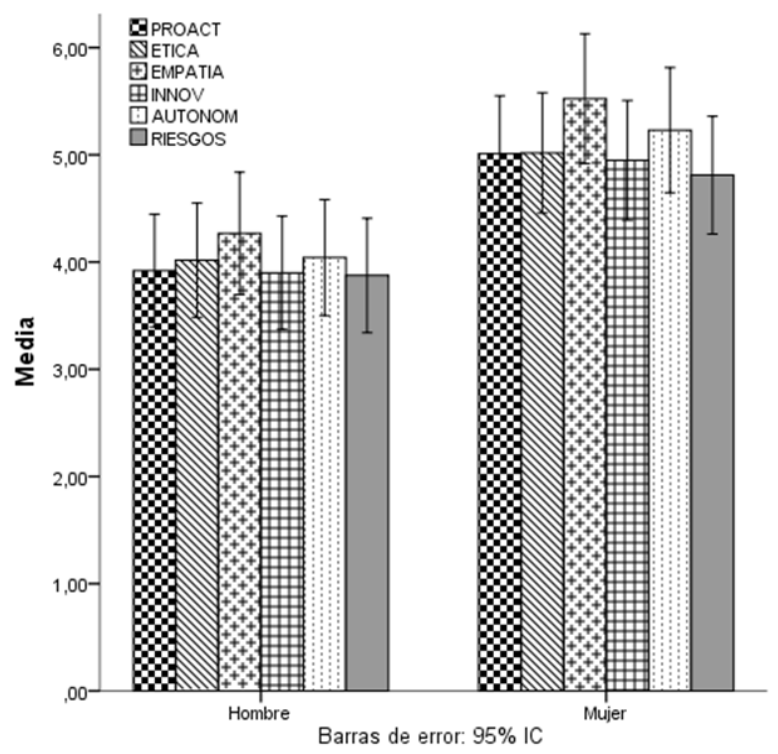

Figura 2. Medias de las dimensiones de la escala de actitudes emprendedoras en educadores Fuente: Elaboración propia.

\section{Discusión}

Para el contexto educativo actual, la educación en emprendimiento es una prioridad, como motor fundamental de crecimiento y desarrollo. Así pues, considerando el emprendimiento como una práctica clave para reactivar los sistemas económicos e impulsar un crecimiento más sostenible y coherente, su incorporación al sistema educativo debería ser inmediata. Para dicha incorporación, resultará esencial conocer los recursos humanos con que se cuenta, esto es, poder evaluar las actitudes emprendedoras del profesorado. Hasta la fecha no constan herramientas diagnósticas a tal efecto en la literatura internacional revisada. En este escenario, el objetivo es disponer de un nuevo instrumento de evaluación de actitudes emprendedoras para profesores, consistente en la adaptación de uno breve y fiable recientemente desarrollado y validado en estudiantes, aportando evidencias de su comportamiento psicométrico en una aplicación al contexto educativo español.

Para conseguir este objetivo, se han llevado a cabo diversos análisis estadísticos. El primero de ellos, un análisis factorial confirmatorio, pone a prueba la estructura de 6 factores, basada en la evidencia a priori ofrecida en Oliver y Galiana (2015), en el que se presentaba la Escala de Actitudes Emprendedoras para Estudiantes. Los resultados del modelo de ecuaciones estructurales fueron muy satisfactorios, ofreciendo evidencia de un ajuste general y analítico adecuado, con saturaciones elevadas en todos los ítems de la escala. De esta forma, las actitudes emprendedoras que tradicionalmente se han apuntado en la literatura científica en psicología organizacional y economía (ver, por ejemplo, Dyson, 2001; HermansenKobulnicky y Moss, 2004; Lumpkin y Dess, 1996; Schafermeyer y Hobson, 1997), también están presentes en el contexto de la educación. 
En segundo lugar, las estimaciones de fiabilidad de las diferentes dimensiones del emprendimiento también resultan satisfactorias. Las estimaciones de fiabilidad de la escala por procedimientos clásicos se revelan muy altas a tenor de los rationale de consenso en este ámbito (Cicchetti, 1994; Clark y Watson, 1995). Se trata, pues, de un instrumento de medida fiable y que, por tanto, puede utilizarse en futuras investigaciones centradas en la educación para el emprendimiento, tanto para aproximarnos a la prevalencia de estas actitudes en el profesorado, como para monitorear su evolución allá donde se implementen intervenciones. La educación en emprendimiento para el profesorado es un aspecto decisivo, y la formación de los formadores en emprendimiento es cada vez más necesaria (González-Contreras, 2014).

Finalmente, se estudia el comportamiento diferencial de las actitudes emprendedoras en profesores en relación con la edad, sexo, situación laboral, tipo de centro y nivel educativo. Estos resultados no identifican relación de las actitudes emprendedoras ni con el tipo de centro, ni con nivel educativo ni con el hecho de estar formándose como docente o trabajando como tal. Sin embargo, sí se encuentra relación estadísticamente significativa de signo negativo entre todas las dimensiones del emprendimiento y la edad. Este resultado apunta hacia la conveniencia de investigar si existe un posible decremento de estas actitudes con el paso de los años o quizá estemos ante diferencias generacionales. Esta evidencia al respecto de la edad, coincide con informes del Eurobarómetro 354 (Comisión Europea, 2012b), en que se apunta mayor tendencia al autoempleo en el caso de jóvenes que de mayores. Por otro lado, aunque algunos estudios hayan señalado mayor presencia de hombres emprendedores (Comisión Europea, 2012b) llegando casi a doblar el número de mujeres (Coduras y Justo, 2003; Reynolds, Hay, Bygrave, Camp y Autio, 2000), nuestra investigación arroja diferencias entre hombres y mujeres, con mayores puntuaciones en todas las actitudes emprendedoras para las docentes. La evidencia anterior en estudiantes españoles de secundaria y universidad, mostraba mayor presencia de innovación en los hombres que en las mujeres, pero simultáneamente menor de proactividad y empatía, no encontrándose diferencias estadísticamente significativas en el resto de dimensiones emprendedoras (Oliver, Galiana y Gutiérrez, 2016). Se espera que futuros estudios profundicen en estos matices diferenciales a través de edades y colectivos. En cuanto al componente de género, podríamos estar ante un cambio de rumbo en nuestra forma de abordar el emprendimiento, en el que las mujeres reivindicarían su papel dinamizador en la sociedad.

A pesar de limitaciones de este estudio, comola naturaleza del muestreo en el que no se pudo obtener la tasa de respuesta, que restringe la generalización de los resultados, el presente trabajo cuenta también con fortalezas. En primer lugar, se trata de un instrumento riguroso para evaluar las actitudes emprendedoras en educadores, profesionales llamados a ser los promotores de la nueva sociedad emprendedora. Además, los resultados ofrecen evidencia de adecuadas propiedades psicométricas, de forma que en el futuro pueda ser usada tanto en un contexto de investigación orientado al autoempleo y la inserción laboral como en monitorización de medidas e intervenciones educativas, sirviendo como criterio para evaluar acciones de promoción del emprendimiento. Finalmente, los resultados relativos a la edad y al género, apuntan el mantenimiento de la tendencia del emprendimiento a decrecer con el paso de los años, mientras que remarcan mayores actitudes emprendedoras en las mujeres. De aportarse más evidencia en esta línea, el diseño de programas futuros centrados en el fomento del emprendimiento debería concentrar sus 
esfuerzos en ciertas franjas de edad. Podrían crearse programas específicos para estimular el emprendimiento en personas mayores. Un docente mayor con una buena formación en emprendimiento, podría alentar el espíritu emprendedor de sus estudiantes incluso sin dominar las nuevas tecnologías que pueden ser un hándicap en ciertas cohortes de edad. Docentes de todas las edades podrían fomentar el espíritu emprendedor de sus estudiantes con una adecuada sensibilización y formación: ambos colectivos (discentes y docentes) saldrían beneficiados. En el caso de los docentes de mayor edad, incluso podría revertir en satisfacción y bienestar psicológico. Si pensamos en las últimas etapas de desarrollo psicosocial de Erikson relacionado con la generatividad, se podría traducir en un mayor interés por formar jóvenes para ser capaces de pensar «fuera de la caja».

A la vista de los datos, el emprendimiento ya no sería una cuestión de género, la sociedad habría avanzado también en este ámbito de la igualdad. Próximos trabajos podrían estudiar la relación entre la presencia de actitudes emprendedoras en los educadores y cómo éstas revierten en una mayor presencia de actitudes emprendedoras en los alumnos. El magnífico trabajo de Ureña (2015), al estudiar la calidad docente universitaria dominicana, recoge de Garbarini (2011) que la "misión fundamental de la docencia es ayudar al alumno a formular su proyecto de vida, y a fortalecer su voluntad, de modo que sea capaz de llevarlo a término". A cuantificar en qué medida nuestras actitudes se orientan a este objetivo, va precisamente destinada la aportación de este trabajo. En definitiva, en el panorama educativo actual, parecía esencial contar con una herramienta que permitiera explorar las actitudes de los educadores y profesores en cuanto a actitudes emprendedoras. Usando esta herramienta para identificar los docentes más emprendedores, se puede reforzar su papel en el organigrama de los centros, permitiéndoles un mayor rango de competencias de orientación educativa. Este incremento de funciones debería ir parejo a un mayor respaldo de la administración, facilitando los recursos para ello. Al fin y al cabo, una de las claves de la innovación es optimizar los recursos que ya se tienen, gestionándolos como no se había hecho antes. Muchas son las respuestas que podríamos dar a la pregunta de por qué medir el emprendimiento en educadores. Quizá la primera sea por su potencial para mejorar la calidad del proceso de enseñanza-aprendizaje.

\section{Referencias}

Akyurek, C., y Sahin, C. (2013). Evaluation of entrepreneurship skills of elementary teachers. EKEV Academic Journal, 17, 51-68.

Atali, L., y Sertbas, K. (2013). A research on individual innovativeness levels of football referees (Kocaeli region case). Turkish Journal of Sport and Exercise, 15, 18-21.
Bayraktar, D. M. (2012). Adoption of web 2.0 tools and the individual innovativeness levels of instructors. Journal of Hasan Ali Yucel Education Faculty, 18, 35-47.

Blenker, P., Elmholdt, S. T., Frederiksen, S. H., Korsgaard, S., y Wagner, K. (2014). Methods in entrepreneurship education research: a review and integrative framework. Education \& Training, 56, 697-715. 
Bonnarens, J. K. (1999). Determining the level of patient care specialty service development and entrepreneurial characteristics present in independent community pharmacy [master thesis]. University of Mississippi, Oxford, 1999.

Borasi, R., y Finnigan, K. (2010). Entrepreneurial attitudes and behaviors that can help prepare successful change-agents in education. The New Educator, 6, 1-29.

Celik, K. (2013). The relationship between individual innovativeness and selfefficacy levels of student teachers. International Journal of Scientific Research in Education, 6, 56-67.

Cicchetti, D. V. (1994). Guidelines, criteria, and rules of thumb for evaluating normed and standardized assessment instruments in psychology. Psychological Assessment, 6, 284290.

Clark, L. A., y Watson, D. (1995). Constructing validity: Basic issues in objective scale development. Psychological Assessment, 7, 309-319.

Coduras, A. y Justo, R. (2003). Global Entrepreneurship Monitor (GEM). Informe ejecutivo 2002. España. Madrid: Instituto de Empresa.

Cohen, J. (1992). A power premier. Psychological Bulletin, 112, 155-159.

Comisión Europea. (2012a). Entrepreneurship Education at School in Europe National Strategies, Curricula and Learning Outcomes. Recuperado de: http://eacea.ec.europa.eu/ education/eurydice/documents/ thematic reports/135EN.pdf.

Comisión Europea. (2012b). Flash Eurobarometer 354. Entrepreneurship in the EU and beyond. Recuperado de: http://ec.europa.eu/public opinion/ flash/fl 354 en.pdf.

Dyson, C. (2001). What makes a great entrepreneur? European Business Review, 13(5), 307-312.

Eraslan, L. (2011). Entrepreneurship teaching at primary education curriculum (sample of life science lesson). The Journal of The Industrial Arts Education Faculty of Gazi University, 27, 82-94.

Evans, D., y Jovanovic, B. (1989). An estimated model of entrepreneurial choice under liquidity constraints. Journal of Political Economy, 97, 808-827.

Evans, D., y Jovanovic, B., (1989). An estimated model of entrepreneurial choice under liquidity constraints. Journal of Political Economy, 97(4), 808-827.

Eyal, O., y Yosef-Hassidim, D. (2012). Managing educational champions: entrepreneurship in schools. Journal of School Leadership, 22, 210-255.

Garbarini, M. (2011). La personalidad del docente. Su influencia en el alumnado. Disponible en: http://fido.palermo. edu/servicios dyc/encuentro2010/ administracion-concursos/archivos conf 2012/496 51611 554con.pdf.

Gibb, A., y Hannon, P. (2006). Towards the entrepreneurialuniversity? International Journal of Entrepreneurship Education, 4, 73-110.

González-Contreras, T. (2014). La importancia de la Innovación y el Emprendimiento en los docentes del Sistema Educacional Chileno. Revista Gestión de las Personas y Tecnología, 19. 
Gur-Erdogan, D.,Eksioglu,S., Zafer-Gunes, D., y Sezen-Gultekin, G. (2014). The Relationship between Social Entrepreneurship Characteristics and the Personal Innovativeness of Prospective Teachers. Anthropologist, 18, 727-733.

Gur-Erdogan, D.,y Zafer-Gunes, D. (2013). The relationship between individual innovativeness and change readiness conditions of students attending faculty of education. Procedia-Social and Behavior Sciences, 106, 30333040.

Hermansen-Kobulnicky, C. J., \& Moss, C. L. (2004). Pharmacy student entrepreneurial orientation: a measure to identify potential pharmacist entrepreneurs. American Journal of Pharmaceutical Education, 68(5). Recuperado de http:// archive.ajpe.org/aj6805/aj6805113/ aj6805113.pdf.

Hu, L., y Bentler, P. M. (1999). Cut-off criteria for fit indexes in covariance structure analysis: Conventional criteria versus new alternatives. Structural Equation Modeling, 6, 1-55.

Kline, R. B. (1998). Principles and practice of structural equation modeling. New York, NY: Guilford Press.

Leisey, D., y Lavaroni, C. (2000). The educational entrepreneur: making a difference. San Rafael, CA: Edupreneur Press.

Lumpkin, G. T., y Dess, G. G. (1996). Clarifying the entrepreneurial orientation construct and linking it to performance. Academy of Management Review, 21, 135-172.

Mars, M., y Rios-Aguilar, C. (2010). Entrepreneurship (re)defined: significance and implications for the scholarship of higher education. Higher Education, 59, 441-460.
Miller, D. (1983). The correlates of entrepreneurship in three types of firms. Management Science, 29, 770-791.

Miner, J. B. (1997). The expanded horizon for achieving entrepreneurial success. Organizational Dynamics, 25(3), 54-67.

OECD. (2009). Evaluation of programs concerning education for entrepreneurship. Paris: OECD Working Party on SME's and Entrepreneurship.

Oliver, A., Galiana, L., y Gutiérrez, M. (2016). Políticas de promoción y diagnóstico del emprendimiento en estudiantes. Anales de Psicología, 32, 183-189.

Oliver, A., y Galiana, L. (2015). Development and validation of the Entrepreneurial Attitudes Scale for Students (EASS). The Spanish Journal of Psychology, 18, E4.

Pittaway, L., y Cope, J. (2007). Entrepreneurship education. International Small Business Journal, 25, 479-510.

Puri, M., y Robinson, D. (2005). Optimism, entrepreneurship, and economic choice. NBER Working Paper.

Reynolds, P. D., Hay, M., Bygrave, W. D., Camp, S. M. y Autio, E. (2000). Global Entrepreneurship Monitor. 2000 executive report. Recuperado de: http://www.gemconsortium.org/ docs/download/2408.

Sánchez, I., Benavides, M. M., y Luna, R. (2004). El proceso de aprendizaje para los emprendedores en la situación actual: un análisis cualitativo en el ámbito universitario. Revista de dirección, organización y administración de empresas, 30, 3448. 
Schafermeyer, K. W., y Hobson, E. H. (1997). Business plans: tool to teach entrepreneurial and communication skills. American Association of Colleges of Pharmacy Annual Meeting, $95,81$.

Steiger, J. H., y Lind, C. (1980). Statistically based tests for the number of common factors. Paper presented at the annual meeting of the Psychometric Society, Iowa City, IA.

Tabachnick, G. G., y Fidell, L. S. (2007). Using multivariate statistics (6a Edición). Boston: Allyn and Bacon.
Tanaka J. S. (1993). Multifaceted conceptions of fit in structural equation models. En K. A. Bollen y J. S. Long (Ed.), Testing structural equation models (pp. 10-39). Newbury Park, CA: Sage.

Teske, P., y Williamson, A. (2006). Entrepreneurs at work. En F. M. Hess (Ed.), Educational entrepreneurship: realities, challenges, possibilities, Harvard Education Press (pp. 45-62). Cambridge: MA.

Ureña, A. J. (2015). Factores relevantes de la calidad de vida docente en profesores de la Universidad Autónoma de Santo Domingo: Un estudio exploratorio. Tesis doctoral. Universitat de València.

Anexo. Escala de actitudes emprendedoras en educadores.

\begin{tabular}{|c|c|c|}
\hline Dimensión & Ítem & Contenido del ítem \\
\hline \multirow[t]{3}{*}{ Proactividad } & 1 & $\begin{array}{l}\text { Planear oportunidades futuras tras finalizar mis estudios ha sido, es o será una parte importante de mi } \\
\text { formación. }\end{array}$ \\
\hline & 2 & $\begin{array}{l}\text { Sabiendo que las condiciones en mi profesión están cambiando, intento buscar activamente nuevas } \\
\text { oportunidades. }\end{array}$ \\
\hline & 3 & Quiero tener lo necesario para avanzar o ser pionero en mi campo profesional. \\
\hline \multirow[t]{3}{*}{ Ética profesional } & 4 & Imagino que seré/Me considero muy ambicioso en mi trabajo. \\
\hline & 5 & Me gusta la idea de tener retos en mi práctica profesional. \\
\hline & 6 & Me considero alguien con alta motivación en el trabajo. \\
\hline \multirow[t]{3}{*}{ Empatía } & 7 & Tengo un gran deseo de ayudar a los demás. \\
\hline & 8 & Creo que hacer una contribución a la sociedad es importante. \\
\hline & 9 & $\begin{array}{l}\text { Soy/seré bueno empatizando con mis alumnos, compañeros, directores, inspectores porque soy receptivo a } \\
\text { sus problemas. }\end{array}$ \\
\hline \multirow[t]{3}{*}{ Innovación } & 10 & Me puedo imaginar hacienda algo innovador como profesional. \\
\hline & 11 & $\begin{array}{l}\text { Creo que un día tendré las habilidades necesarias para desarrollar un nuevo método formativo o una } \\
\text { programación innovadora para mejorar el aprendizaje de los alumnos. }\end{array}$ \\
\hline & 12 & $\begin{array}{l}\text { Me puedo ver a mí mismo empezando algo innovador en los grupos en los que hago formación o en el lugar } \\
\text { de trabajo. }\end{array}$ \\
\hline \multirow[t]{3}{*}{ Autonomía } & 13 & Como profesional de la educación quiero animarme a desarrollar nuevas ideas en el lugar de trabajo. \\
\hline & 14 & $\begin{array}{l}\text { Quiero trabajar donde las nuevas oportunidades importen a todos los miembros de la organización o la } \\
\text { escuela donde trabaje. }\end{array}$ \\
\hline & 15 & Quiero trabajar donde pueda sugerir nuevas ideas a los que toman las decisiones. \\
\hline \multirow[t]{3}{*}{ Toma de riesgos } & 16 & Creo que soy arriesgado comparado con otros compañeros docentes que conozco. \\
\hline & 17 & $\begin{array}{l}\text { Creo que para ser un docente de éxito tendré que tomar riesgos en mis clases, programaciones, forma de } \\
\text { organizar la docencia, etc. }\end{array}$ \\
\hline & 18 & Prefiero arriesgarme y perder, que darme cuenta más adelante que desperdicié una gran oportunidad. \\
\hline
\end{tabular}

